\title{
Weighted Estimates for Rough Maximal Functions with Applications to Angular Integrability
}

\author{
Feng Liu $\mathbb{D}^{1}{ }^{1}$ and Fangfang $X u \mathbb{C}^{1,2}$ \\ ${ }^{1}$ College of Mathematics and Systems Science, Shandong University of Science and Technology, Qingdao, Shandong 266590, China \\ ${ }^{2}$ School of Automation, Qingdao University, Qingdao 266590, China
}

Correspondence should be addressed to Feng Liu; liufeng860314@163.com

Received 7 May 2019; Accepted 1 July 2019; Published 16 July 2019

Academic Editor: Huy Qui Bui

Copyright (c) 2019 Feng Liu and Fangfang Xu. This is an open access article distributed under the Creative Commons Attribution License, which permits unrestricted use, distribution, and reproduction in any medium, provided the original work is properly cited.

In this note we establish certain weighted estimates for a class of maximal functions with rough kernels along "polynomial curves" on $\mathbb{R}^{n}$. As applications, we obtain the bounds of the above operators on the mixed radial-angular spaces, on the vector-valued mixed radial-angular spaces, and on the vector-valued function spaces. Particularly, the above bounds are independent of the coefficients of the polynomials in the definition of the operators.

\section{Introduction}

During the last several years, a considerable amount of attention has been given to the investigation of the boundedness for various kinds of integral operators on the Lebesgue spaces and other more general function spaces (see [1-8], for examples). The primary aim of this article is to establish the boundedness for maximal operators related to singular integrals on the mixed radial-angular spaces.

Let $\mathbb{R}^{n}(n \geq 2)$ be the $n$-dimensional Euclidean space and $S^{n-1}$ denote the unit sphere in $\mathbb{R}^{n}$ equipped with the induced Lebesgue measure $d \sigma$. Assume that $\Omega \in L^{1}\left(S^{n-1}\right)$ is a homogeneous function of degree zero and satisfies

$$
\int_{S^{n-1}} \Omega(u) d \sigma(u)=0 .
$$

Let $P_{N}(t)$ be a real polynomial on $\mathbb{R}$ of degree $N$ satisfying $P_{N}(0)=0$ and $h$ be a suitable function defined on $\mathbb{R}$; we define the singular integral operator $T_{h, \Omega, P_{N}}$ along the "polynomial curve" $P_{N}$ on $\mathbb{R}^{n}$ by

$$
\begin{aligned}
& T_{h, \Omega, P_{N}} f(x) \\
& \quad=\text { p.v. } \int_{\mathbb{R}^{n}} f\left(x-P_{N}(|y|) y^{\prime}\right) \frac{h(|y|) \Omega\left(y^{\prime}\right)}{|y|^{n}} d y,
\end{aligned}
$$

where $y^{\prime}=y /|y|$ for $y \neq 0$. Let $\mathbb{R}_{+}:=(0, \infty)$ and $\mathscr{H}_{\gamma}\left(\mathbb{R}_{+}\right)(\gamma>0)$ denote the set of all measurable functions $h: \mathbb{R}_{+} \longrightarrow \mathbb{R}$ satisfying

$$
\|h\|_{\mathscr{H}_{\gamma}\left(\mathbb{R}_{+}\right)}:=\left(\int_{0}^{\infty}|h(t)|^{\gamma} \frac{d t}{t}\right)^{1 / \gamma}<\infty .
$$

Let $T_{h, \Omega, P_{N}}$ be defined as in (2) and let $\bar{U}\left(\mathbb{R}_{+}\right)$denote the closed unit ball in $\mathscr{H}_{2}\left(\mathbb{R}_{+}\right)$. Define the maximal function $\mathcal{S}_{\Omega, P_{N}}$ by

$$
\mathcal{S}_{\Omega, P_{N}} f(x)=\sup _{h \in \bar{U}\left(\mathbb{R}_{+}\right)}\left|T_{h, \Omega, P_{N}} f(x)\right| .
$$

If $P_{N}(t)=t$ and $h(t) \equiv 1$, we denote $T_{h, \Omega, P_{N}}=T_{\Omega}$ and $\mathcal{S}_{\Omega, P_{N}}=\mathcal{S}_{\Omega}$.

As a formal extension of the Lebesgue space $L^{p}$, the mixed radial-angular space $L_{|x|}^{p} L_{\theta}^{\tilde{p}}\left(\mathbb{R}^{n}\right)$ has already been successfully used in studying Strichartz estimates and dispersive equations (see [9-16]) over the last several years. Recall that the mixed radial-angular spaces $L_{|x|}^{p} L_{\theta}^{\tilde{p}}\left(\mathbb{R}^{n}\right), 1 \leq p, \widetilde{p} \leq \infty$, consist of all functions $u$ satisfying $\|u\|_{L_{|x|}^{p} L_{\theta}^{\tilde{p}}\left(\mathbb{R}^{n}\right)}<\infty$, where

$$
\|u\|_{L_{|x|}^{p} L_{\theta}^{\tilde{p}}\left(\mathbb{R}^{n}\right)}:=\left(\int_{0}^{\infty}\|u(\rho \cdot)\|_{L^{\tilde{p}}\left(S^{n-1}\right)}^{p} \rho^{n-1} d \rho\right)^{1 / p}
$$


and

$$
\|u\|_{L_{|x|}^{\infty} \tilde{L}_{\theta}^{\tilde{p}}\left(\mathbb{R}^{n}\right)}:=\sup _{\rho>0}\|u(\rho \cdot)\|_{L^{\tilde{p}}\left(S^{n-1}\right)} \cdot
$$

It is clear that the spaces $L_{|x|}^{p} L_{\theta}^{\widetilde{p}}\left(\mathbb{R}^{n}\right)$ have the following easy properties.

(i) If $1 \leq p \leq \infty$ and $\widetilde{p}=p$, then

$$
\|u\|_{L_{|x|}^{p} L_{\theta}^{\tilde{p}}\left(\mathbb{R}^{n}\right)}=\|u\|_{L^{p}\left(\mathbb{R}^{n}\right)}
$$

(ii) If $u$ is a radial function on $\mathbb{R}^{n}$ and $1 \leq p \leq \infty, 1 \leq$ $\widetilde{p} \leq \infty$, then

$$
\|u\|_{L_{|x|}^{p} L_{\theta}^{\tilde{p}}\left(\mathbb{R}^{n}\right)} \simeq\|u\|_{L^{p}\left(\mathbb{R}^{n}\right)} .
$$

(iii) If $1 \leq p \leq \infty$ and $1 \leq \widetilde{p}_{1} \leq \widetilde{p}_{2} \leq \infty$, then

$$
\|u\|_{L_{|x|}^{p} \tilde{L}_{\theta}^{\tilde{p}_{1}}\left(\mathbb{R}^{n}\right)} \leq C_{n, p, \widetilde{p}_{1}, \widetilde{p}_{2}}\|u\|_{L_{|x|}^{p} \tilde{L}_{\theta}^{\tilde{p}_{2}}\left(\mathbb{R}^{n}\right)}
$$

Here the notation $A \simeq B$ means that there are two positive constants $C, C^{\prime}$ such that $A \leq C B$ and $B \leq C^{\prime} A$. Throughout this paper, we use $C_{\alpha, \beta, \ldots}$ to denote positive constants that depend on parameters $\alpha, \beta, \ldots$.

Recently, the mixed radial-angular space $L_{|x|}^{p} L_{\theta}^{\widetilde{p}}\left(\mathbb{R}^{n}\right)$ is also playing active roles in the theory of singular integral operator (see [17-19]). In [18], among other things, Córdoba proved that $T_{\Omega}$ is bounded on $L_{|x|}^{p} L_{\theta}^{\widetilde{p}}\left(\mathbb{R}^{n}\right)$ for all $1<p<$ $\infty$ and $\tilde{p}=2$, provided that $\Omega \in \mathscr{C}^{1}\left(S^{n-1}\right)$. Later on, D'Ancona and Lucà [19] extended the above index $\widetilde{p}=2$ to the range $1<\widetilde{p}<\infty$ by applying the same argument in [18, Theorem 2.1] (see also [17, Theorem 1.1] for the weighted case). Recently, Liu and Fan [20] improved the above result to the case $\Omega \in L^{q}\left(S^{n-1}\right)$ and extended the above results to the singular integral operators along polynomial curves. Precisely, let $\Delta_{\gamma}\left(\mathbb{R}_{+}\right)(\gamma>0)$ be the set of all measurable functions $h$ defined on $\mathbb{R}_{+}$satisfying

$$
\|h\|_{\Delta_{\gamma}\left(\mathbb{R}_{+}\right)}:=\sup _{R>0}\left(R^{-1} \int_{0}^{R}|h(t)|^{\gamma} d t\right)^{1 / \gamma}<\infty .
$$

Liu and Fan [20] proved the following result. Our main results can be formulated as follows.

Theorem A (see [20]). Let $P_{N}(t)$ be a real polynomial on $\mathbb{R}$ of degree $N$ and satisfy $P_{N}(0)=0$. Suppose that $\Omega \in L^{q}\left(S^{n-1}\right)$ satisfies (1) and $h \in \Delta_{\gamma}\left(\mathbb{R}_{+}\right)$for some $1<q, \gamma \leq \infty$. Then for $1<p, \widetilde{p}<\infty$, the following inequalities hold:

$$
\begin{gathered}
\left\|T_{h, \Omega, P_{N}} f\right\|_{L_{|x|}^{p} L_{\theta}^{\tilde{p}}\left(\mathbb{R}^{n}\right)} \leq C_{h, \Omega, q, \gamma, p, \tilde{p}, N}\|f\|_{L_{|x|}^{p} L_{\theta}^{\tilde{p}}\left(\mathbb{R}^{n}\right)} ; \\
\left\|\left(\sum_{j \in \mathbb{Z}}\left|T_{h, \Omega, P_{N}} f_{j}\right|^{\tilde{p}}\right)^{1 / \tilde{p}}\right\| \\
\leq C_{h, \Omega, q, \gamma, p, \tilde{p}, N}\left\|\left(\sum_{L_{|x|}^{p} L_{\theta}^{\tilde{p}}\left(\mathbb{R}^{n}\right)}\left|f_{j}\right|^{\tilde{p}}\right)^{1 / \tilde{p}}\right\|_{L_{|x|}^{p} \tilde{L}_{\theta}^{\tilde{p}}\left(\mathbb{R}^{n}\right)} \\
\left\|\left(\sum_{j \in \mathbb{Z}}\left|T_{h, \Omega, P_{N}} f_{j}\right|^{\tilde{p}}\right)^{1 / \tilde{p} \|}\right\|_{L^{p}\left(\mathbb{R}^{n}\right)}\left\|\left(\sum_{j \in \mathbb{Z}}\left|f_{j}\right|^{\tilde{p}}\right)^{1 / \tilde{p}}\right\|_{L^{p}\left(\mathbb{R}^{n}\right)} .
\end{gathered}
$$

Here the above constants $C_{h, \Omega, q, \gamma, p, \tilde{p}, N}>0$ are independent of the coefficients of $P_{N}$.

On the other hand, the classical maximal operator $\mathcal{S}_{\Omega}$ was originally introduced by Chen and Lin [21] who proved that if $\Omega \in \mathscr{C}^{1}\left(S^{n-1}\right)$, then $\mathcal{S}_{\Omega}$ is of type $(p, p)$ for any $p>2 n /(2 n-1)$ and the range of $p$ is best possible. Subsequently, the $L^{p}$ mapping properties of $\mathcal{S}_{\Omega}$ have been discussed extensively by many authors (see [22-26], for example). Particularly, AlSalman [23] proved the following result.

Theorem B (see [23]). Let $\Omega$ satisfy (1) and $\Omega \in$ $L(\log L)^{1 / 2}\left(S^{n-1}\right)$. Then

$$
\left\|\mathcal{S}_{\Omega, P_{N}} f\right\|_{L^{p}\left(\mathbb{R}^{n}\right)} \leq C_{p}\|f\|_{L^{p}\left(\mathbb{R}^{n}\right)}
$$

for all $2 \leq p<\infty$. Here $C_{p}>0$ is independent of the coefficients of $P_{N}$.

It is well known that the following are valid.

$$
\begin{aligned}
& \mathscr{H}_{\infty}\left(\mathbb{R}_{+}\right)=\Delta_{\infty}\left(\mathbb{R}_{+}\right)=L^{\infty}\left(\mathbb{R}_{+}\right) ; \\
& \Delta_{\gamma_{1}}\left(\mathbb{R}_{+}\right) \subsetneq \Delta_{\gamma_{2}}\left(\mathbb{R}_{+}\right), \quad \forall 1<\gamma_{2}<\gamma_{1}<\infty ; \\
& \mathscr{H}_{\gamma}\left(\mathbb{R}_{+}\right) \subsetneq \Delta_{\gamma}\left(\mathbb{R}_{+}\right), \quad \forall 1<\gamma<\infty ; \\
& \mathscr{C}^{1}\left(S^{n-1}\right) \subsetneq \bigcup_{1<q \leq \infty} L^{q}\left(S^{n-1}\right) \subsetneq L(\log L)^{1 / 2}\left(S^{n-1}\right) .
\end{aligned}
$$

It follows from (14) and Theorem B that the condition $\Omega \epsilon$ $L^{q}\left(S^{n-1}\right)$ implies the $L^{p}$-boundedness of $\mathcal{S}_{\Omega, P_{N}}$ for $2 \leq p<\infty$. A question that arises naturally is the following.

Question $A$. Is the operator $\mathcal{S}_{\Omega, P_{N}}$ bounded on $L_{|x|}^{p} L_{\theta}^{\tilde{p}}\left(\mathbb{R}^{n}\right)$ for $1<p, \widetilde{p}<\infty(p \neq \tilde{p})$ if $\Omega \in L^{q}\left(S^{n-1}\right)$ for some $q>1$ ?

In this paper we will give an affirmative answer to Question A. In order to obtain the $L_{|x|}^{p} L_{\theta}^{\widetilde{p}}\left(\mathbb{R}^{n}\right)$ boundedness 
for $\mathcal{S}_{\Omega, P_{N}}$, we shall establish the following weighted estimates for $\mathcal{S}_{\Omega, P_{N}}$.

Theorem 1. Let $P_{N}(t)$ be a real polynomial on $\mathbb{R}$ of degree $N$ and satisfy $P_{N}(0)=0$. Suppose that $\Omega$ satisfies (1) and $\Omega \in L^{q}\left(S^{n-1}\right)$ for some $1<q \leq \infty$. Then, for any nonnegative measurable function $u$ on $\mathbb{R}^{n}$, it holds that

$$
\left\|\mathcal{S}_{\Omega, P_{N}} f\right\|_{L^{p}(u)} \leq C_{\Omega, q, p, s, N}\|f\|_{L^{p}\left(\Lambda_{N, s} u\right)},
$$

provided that one of the following conditions holds:

(i) $1<p<2$ and $s>2 / p$;

(ii) $2 \leq p<\infty$ and $s>1$.

Here $\Lambda_{N, s} u=\sum_{i=0}^{N} \mathrm{M}_{s}^{N+1-i} M_{i, s}^{\widetilde{\sigma}} \mathrm{M}_{s} u$ and $M_{\lambda, s}^{\widetilde{\sigma}} u=\left(M_{\lambda}^{\tilde{\sigma}}\left(u^{s}\right)\right)^{1 / s}$, $\mathrm{M}_{s}^{k} u=\left(\mathrm{M}^{k} u^{s}\right)^{1 / s}$ for any $k \in \mathbb{N}$. $\mathrm{M}^{k}$ is the Hardy-Littlewood maximal operator $\mathrm{M}$ iterated $k$ times for all $k=1,2, \cdots$. Specially, $\mathrm{M}^{k}=\mathrm{M}$ when $k=1 . \mathrm{M}_{\lambda}^{\widetilde{\sigma}}$ is a maximal operator defined as in Section 2. result.

As applications of Theorem 1, we can obtain the following

Theorem 2. Let $\mathcal{S}_{\Omega, P_{N}}$ be the maximal operator defined by (4). Let $P_{N}(t)$ be a real polynomial on $\mathbb{R}$ of degree $N$ and satisfy $P_{N}(0)=0$. Suppose that $\Omega$ satisfies (1) and $\Omega \in L^{q}\left(S^{n-1}\right)$ for some $1<q \leq \infty$. Then, for $1<\tilde{p}<2$ and $\widetilde{p}<p<2 \widetilde{p} /(2-\widetilde{p})$ or $2 \leq \widetilde{p} \leq p<\infty$, the following inequalities hold:

$$
\begin{aligned}
& \left\|\mathcal{S}_{\Omega, P_{N}} f\right\|_{L_{|x|}^{p} L_{\theta}^{\tilde{p}}\left(\mathbb{R}^{n}\right)} \leq C_{\Omega, q, p, \tilde{p}, N}\|f\|_{L_{|x|}^{p} L_{\theta}^{\tilde{p}}\left(\mathbb{R}^{n}\right)} \\
& \left\|\left(\sum_{j \in \mathbb{Z}}\left|\mathcal{S}_{\Omega, P_{N}} f_{j}\right|^{\tilde{p}}\right)^{1 / \tilde{p}}\right\| \\
& \leq C_{\Omega, q, p, \tilde{p}, N}\left\|\left(\sum_{L_{|x|}^{p} L_{\theta}^{\tilde{p}}\left(\mathbb{R}^{n}\right)}\left|f_{j}\right|^{\tilde{p}}\right)^{1 / \tilde{p}}\right\|_{L_{|x|}^{p} L_{\theta}^{\tilde{p}}\left(\mathbb{R}^{n}\right)} ; \\
& \left\|\left(\sum_{j \in \mathbb{Z}}\left|\mathcal{S}_{\Omega, P_{N}} f_{j}\right|^{\tilde{p}}\right)^{1 / \tilde{p}}\right\|\left\|_{L^{p}\left(\mathbb{R}^{n}\right)}\right\|\left(\sum_{j \in \mathbb{Z}}\left|f_{j}\right|^{\tilde{p}}\right)^{1 / \tilde{p}} \|_{L^{p}\left(\mathbb{R}^{n}\right)}
\end{aligned}
$$

Here the constants $C_{\Omega, q, p, \tilde{p}, N}>0$ are independent of the coefficients of $P_{N}$.

Remark 3. We remark that our main results are new even in the special case $P_{N}(t)=t$.

The rest of this paper is organized as follows. In Section 2 we shall present some notations and auxiliary lemmas. The proofs of Theorems 1 and 2 will be given in Section 3. It should be pointed out that the main idea in the proof of Theorem 1 is a combination of ideas and arguments from [18-20, 27-29]. The proof of Theorem 2 is based on Theorem 1 and a criterion established in Section 2 (see Proposition 7). Throughout this note, for any $p \in(1, \infty)$, we let $p^{\prime}$ denote the dual exponent to $p$ defined as $1 / p+1 / p^{\prime}=1$. We also use the convention $\prod_{j \in \emptyset} a_{j}=1$. In what follows, for any function $f$, we define $\tilde{f}$ by $\tilde{f}(x)=f(-x)$. For $f \in L^{p}(w)$, we set

$$
\|f\|_{L^{p}(w)}=\left(\int_{\mathbb{R}^{n}}|f(x)|^{p} w(x) d x\right)^{1 / p} .
$$

\section{Preliminary Lemmas}

In this section we shall give some notations and necessary lemmas, which will play key roles in the proof of our main results. In what follows, we assume that $P_{N}(t)=\sum_{i=1}^{N} a_{i} t^{i}$ with $a_{i} \neq 0$. Let $P_{\lambda}(t)=\sum_{i=1}^{\lambda} a_{i} t^{i}$ for $\lambda=1,2, \ldots, N$ and $P_{0}(t)=0$. For $\lambda \in\{1,2, \ldots, N\}$, we define a family of measures $\left\{\sigma_{\lambda, r}\right\}_{r>0}$ and the related maximal operator $M_{\lambda}^{\sigma}$ by

$$
\begin{aligned}
\widehat{\sigma_{\lambda, r}}(x) & =\int_{S^{n-1}} e^{-2 \pi i P_{\lambda}(r) x \cdot y^{\prime}} \Omega\left(y^{\prime}\right) d \sigma\left(y^{\prime}\right), \\
M_{\lambda}^{\sigma} f(x) & =\sup _{k \in \mathbb{Z}} \int_{2^{k}}^{2^{k+1}}|| \sigma_{\lambda, r}|* f(x)| \frac{d r}{r},
\end{aligned}
$$

where $\left|\sigma_{\lambda, r}\right|$ is defined in the same way as $\sigma_{\lambda, r}$, but with $\Omega$ replaced by $|\Omega|$. We also define the maximal operator $M_{\lambda}^{\widetilde{\sigma}}$ by

$$
M_{\lambda}^{\widetilde{\sigma}} f(x)=\sup _{k \in \mathbb{Z}} \int_{2^{k}}^{2^{k+1}}|| \widetilde{\sigma}_{\lambda, r}|* f(x)| \frac{d r}{r},
$$

where $\widetilde{\sigma}_{\lambda, r}$ is defined in the following way

$$
\int_{\mathbb{R}^{n}} f(x) d\left|\widetilde{\sigma}_{\lambda, r}\right|(x)=\int_{\mathbb{R}^{n}} f(-x) d\left|\sigma_{\lambda, r}\right|(x) .
$$

Lemma 4 ([30, p.186, corollary $])$. Let $\Phi(t)=t^{\alpha_{1}}+\mu_{2} t^{\alpha_{2}}+\cdots+$ $\mu_{n} t^{\alpha_{n}}$ and $\Psi \in C^{1}[a, b]$, where $\mu_{2}, \ldots, \mu_{n}$ are real parameters and $\alpha_{1}, \ldots, \alpha_{n}$ are distinct positive (not necessarily integer) exponents. Then

$$
\begin{aligned}
& \left|\int_{a}^{b} e^{-i \lambda \Phi(t)} \Psi(t) d t\right| \\
& \quad \leq C \lambda^{-\epsilon}\left\{\sup _{t \in[a, b]}|\Psi(t)|+\int_{a}^{b}\left|\Psi^{\prime}(t)\right| d t\right\},
\end{aligned}
$$

with $\epsilon=\min \left\{1 / \alpha_{1}, 1 / n\right\}$ and $C$ does not depend on $\mu_{2}, \ldots, \mu_{n}$ as long as $0 \leq a<b \leq 1$. 
Lemma 5. Let $\Omega$ satisfy (1) and $\Omega \in L^{q}\left(S^{n-1}\right)$ for some $q>1$. Then for $\lambda \in\{1,2, \ldots, N\}$ and $k \in \mathbb{Z}$, the following estimates hold:

$$
\begin{aligned}
& \sup _{r>0} \max \left\{\left\|\sigma_{\lambda, r}\right\|,\left|\widehat{\sigma_{\lambda, r}}(\xi)\right|,\left|\widehat{\sigma_{\lambda, r}}\right|(\xi) \mid\right\} \mid \leq\|\Omega\|_{L^{1}\left(S^{n-1}\right)} \\
& \int_{2^{k}}^{2^{k+1}}\left|\widehat{\sigma_{\lambda, r}}(\xi)\right|^{2} \frac{d r}{r} \leq C_{\Omega, q} \min \left\{1,\left|2^{k \lambda} a_{\lambda} \xi\right|^{-1 /\left(2 q^{\prime} \lambda\right)}\right\} \\
& \left.\int_{2^{k}}^{2^{k+1}}\left|\widehat{\sigma_{\lambda, r}}\right|(\xi)\right|^{2} \frac{d r}{r} \\
& \leq C_{\Omega, q} \min \left\{1,\left|2^{k \lambda} a_{\lambda} \xi\right|^{-1 /\left(2 q^{\prime} \lambda\right)}\right\}
\end{aligned}
$$

$$
\begin{aligned}
& \int_{2^{k}}^{2^{k+1}}\left|\widehat{\sigma_{\lambda, r}}(\xi)\right|^{2} \frac{d r}{r} \\
& \quad=\int_{2^{k}}^{2^{k+1}} \iint_{\left(S^{n-1}\right)^{2}} \Omega(u) \\
& \quad \overline{\Omega\left(y^{\prime}\right)} e^{-2 \pi i P_{\lambda}(r) \xi \cdot\left(u-y^{\prime}\right)} d \sigma(u) d \sigma\left(y^{\prime}\right) \frac{d t}{t} \\
& \quad \leq \iint_{\left(S^{n-1}\right)^{2}}\left|\Omega(u) \overline{\Omega\left(y^{\prime}\right)}\right|\left|\int_{2^{k}}^{2^{k+1}} e^{-2 \pi i P_{\lambda}(r) \xi \cdot\left(u-y^{\prime}\right)} \frac{d t}{t}\right| d \sigma(u) d \sigma\left(y^{\prime}\right) \\
& \quad \leq \iint_{\left(S^{n-1}\right)^{2}}\left|\Omega(u) \overline{\Omega\left(y^{\prime}\right)}\right|\left|\int_{1}^{2} e^{-2 \pi i P_{\lambda}\left(2^{k} r\right) \xi \cdot\left(u-y^{\prime}\right)} \frac{d t}{t}\right| d \sigma(u) d \sigma\left(y^{\prime}\right) \\
& \quad \leq \iint_{\left(S^{n-1}\right)^{2}}\left|\Omega(u) \overline{\Omega\left(y^{\prime}\right)}\right| \min \left\{1,\left|2^{k \lambda} a_{\lambda} \xi \cdot\left(u-y^{\prime}\right)\right|^{-1 / \lambda}\right\} d \sigma(u) d \sigma\left(y^{\prime}\right) \\
& \quad \leq C\|\Omega\|_{L^{q}\left(S^{n-1}\right)}^{2}\left|2^{k \lambda} a_{\lambda} \xi\right|^{-1 / \epsilon},
\end{aligned}
$$

for any $\epsilon \in\left(0, \min \left\{1 / q^{\prime}, 1 / \lambda\right\}\right)$. This together with (24) yields (25). Similarly, we can prove (26). It is clear that

$$
\begin{aligned}
& \max \left\{\left|\widehat{\sigma_{\lambda, r}}(\xi)-\widehat{\sigma_{\lambda-1, r}}(\xi)\right|,\left|\widehat{\sigma_{\lambda, r}}\right|(\xi)-\widehat{\sigma_{\lambda-1, r}}|(\xi)|\right\} \\
& \leq \int_{S^{n-1}}\left|\Omega\left(y^{\prime}\right)\right|\left|e^{-2 \pi i P_{\lambda}(r) \xi \cdot y^{\prime}}-e^{-2 \pi i P_{\lambda-1}(r) \xi \cdot y^{\prime}}\right| d \sigma\left(y^{\prime}\right) \\
& \leq 2 \pi\|\Omega\|_{L^{1}\left(S^{n-1}\right)}\left|r^{\lambda} a_{\lambda} \xi\right| \leq C_{\Omega, q}\left|r^{\lambda} a_{\lambda} \xi\right|
\end{aligned}
$$

which together with (24) and the fact that $\sigma_{0, r}(\xi)=0$ yields (27)-(28) and completes the proof.

$$
\begin{aligned}
& \int_{2^{k}}^{2^{k+1}}\left|\widehat{\sigma_{\lambda, r}}(\xi)-\widehat{\sigma_{\lambda-1, r}}(\xi)\right|^{2} \frac{d r}{r} \\
& \leq C_{\Omega, q}\left(\min \left\{1,\left|2^{(k+1) \lambda} a_{\lambda} \xi\right|\right\}\right)^{2} ; \\
& \int_{2^{k}}^{2^{k+1}}\left|\widehat{\sigma_{\lambda, r}}\right|(\xi)-\widehat{\sigma_{\lambda-1, r}}|(\xi)|^{2} \frac{d r}{r} \\
& \leq C_{\Omega, q}\left(\min \left\{1,\left|2^{(k+1) \lambda} a_{\lambda} \xi\right|\right\}\right)^{2} .
\end{aligned}
$$

Proof. Estimate (24) is trivial. By Lemma 4, Hölder's inequality, and the changes of variable, we have 
$1\}$ satisfying $\phi(t)=1$ when $|t|<1 / 2$. Define the measures $\left\{\tau_{k}\right\}_{k \in \mathbb{Z}}$ by

$$
\begin{aligned}
\widehat{\tau_{k}}(\xi)= & \int_{2^{k}}^{2^{k+1}} \widehat{\left|\sigma_{l, r}\right|}(\xi) \frac{d r}{r} \\
& -\int_{2^{k}}^{2^{k+1}} \widehat{\left|\sigma_{l-1, r}\right|}(\xi) \frac{d r}{r} \phi\left(\left|2^{k l} a_{l} \xi\right|\right) .
\end{aligned}
$$

By Lemma 5 and the definition of $\tau_{k}$, we have

$$
\begin{gathered}
\left|\widehat{\tau_{k}}(\xi)\right| \leq C_{\Omega, q} \min \left\{1,\left|2^{k \lambda} a_{\lambda} \xi\right|,\left|2^{k \lambda} a_{\lambda} \xi\right|^{-1 /\left(2 q^{\prime} \lambda\right)}\right\} \\
\left\|M_{l}^{\sigma} f\right\|_{L^{p}\left(\mathbb{R}^{n}\right)} \leq\|G(f)\|_{L^{p}\left(\mathbb{R}^{n}\right)}+C\left\|M_{l-1}^{\sigma} f\right\|_{L^{p}\left(\mathbb{R}^{n}\right)}, \\
1<p<\infty \\
\left\|\tau^{*}(f)\right\|_{L^{p}\left(\mathbb{R}^{n}\right)} \leq\|G(f)\|_{L^{p}\left(\mathbb{R}^{n}\right)}+C\left\|M_{l-1}^{\sigma} f\right\|_{L^{p}\left(\mathbb{R}^{n}\right)}, \\
1<p<\infty
\end{gathered}
$$

where

$$
\begin{aligned}
G(f)(x) & =\left(\sum_{k \in \mathbb{Z}}\left|\tau_{k} * f(x)\right|^{2}\right)^{1 / 2} \\
\text { and } \tau^{*}(f)(x) & =\sup _{k \in \mathbb{Z}}|| \tau_{k}|* f(x)|
\end{aligned}
$$

By (34) and Plancherel's theorem, we get

$$
\|G(f)\|_{L^{2}\left(\mathbb{R}^{n}\right)} \leq C_{\Omega, q}\|f\|_{L^{2}\left(\mathbb{R}^{n}\right)} .
$$

From (36)-(38) and our assumption, we have

$$
\left\|\tau^{*}(f)\right\|_{L^{2}\left(\mathbb{R}^{n}\right)} \leq C_{\Omega, q}\|f\|_{L^{2}\left(\mathbb{R}^{n}\right)} .
$$

By the lemma on page 544 of [27] $\left(q=2, p_{0}=4\right)$, we have

$$
\|G(f)\|_{L^{p}\left(\mathbb{R}^{n}\right)} \leq C_{\Omega, q, p}\|f\|_{L^{p}\left(\mathbb{R}^{n}\right)}, \quad \frac{4}{3}<p<4 .
$$

From our assumption, (35) and (40), we get

$$
\left\|\tau^{*}(f)\right\|_{L^{p}\left(\mathbb{R}^{n}\right)} \leq C_{\Omega, q, p}\|f\|_{L^{p}\left(\mathbb{R}^{n}\right)}, \quad \frac{4}{3}<p<4 .
$$

By the lemma on page 544 of [27] $\left(q=4 / 3, p_{0}=8\right)$, we have

$$
\|G(f)\|_{L^{p}\left(\mathbb{R}^{n}\right)} \leq C_{\Omega, q, p}\|f\|_{L^{p}\left(\mathbb{R}^{n}\right)}, \quad \frac{8}{7}<p<8 .
$$

By using this argument repeatedly, one can obtain ultimately

$$
\|G(f)\|_{L^{p}\left(\mathbb{R}^{n}\right)} \leq C_{\Omega, q, p}\|f\|_{L^{p}\left(\mathbb{R}^{n}\right)}, \quad 1<p<\infty .
$$

Combining (43) with (35) and assumption yields that

$$
\left\|M_{l}^{\sigma} f\right\|_{L^{p}\left(\mathbb{R}^{n}\right)} \leq C_{\Omega, q, p}\|f\|_{L^{p}\left(\mathbb{R}^{n}\right)}, \quad 1<p<\infty .
$$

This completes the proof of Lemma 6.
To prove Theorem 1, we need the following proposition, which is of interest in its own right.

Proposition 7. Let $1<p<\infty$ and $s_{0} \geq 1$. Suppose that $T$ is a linear or sublinear operator such that

$$
\|T f\|_{L^{p}(u)} \leq C_{p, s, s_{0}}\|f\|_{L^{p}\left(\mathscr{G}_{s}(u)\right)}, \quad \forall s>s_{0}
$$

for any nonnegative measurable function $u$ on $\mathbb{R}^{n}$, where $\mathscr{G}_{s}$ is a bounded operator from $L^{r}\left(\mathbb{R}^{n}\right)$ to itself for all $r \in(s, \infty)$. Then for any $q \in\left(p, p s_{0} /\left(s_{0}-1\right)\right)$, the following inequalities hold:

$$
\begin{aligned}
& \|T f\|_{L_{|x|}^{q} L_{\theta}^{p}\left(\mathbb{R}^{n}\right)} \leq C_{p, q}\|f\|_{L_{|x|}^{q} L_{\theta}^{p}\left(\mathbb{R}^{n}\right)} ; \\
& \left\|\left(\sum_{j \in \mathbb{Z}}\left|T f_{j}\right|^{p}\right)^{1 / p}\right\|_{L_{|x|}^{q} L_{\theta}^{p}\left(\mathbb{R}^{n}\right)} \\
& \leq C_{p, q}\left\|\left(\sum_{j \in \mathbb{Z}}\left|f_{j}\right|^{p}\right)^{1 / p}\right\|_{L_{|x|}^{q} L_{\theta}^{p}\left(\mathbb{R}^{n}\right)} ;
\end{aligned}
$$

$$
\begin{gathered}
\left\|\left(\sum_{j \in \mathbb{Z}}\left|T f_{j}\right|^{p}\right)^{1 / p}\right\|_{L^{q}\left(\mathbb{R}^{n}\right)} \\
\leq C_{p, q}\left\|\left(\sum_{j \in \mathbb{Z}}\left|f_{j}\right|^{p}\right)^{1 / p}\right\|_{L^{q}\left(\mathbb{R}^{n}\right)} .
\end{gathered}
$$

Proof. We only prove (46) since (47) and (48) can be proved similarly. The argument of the proof for (46) is similar to those of the proof in [19, Theorem 2.6] essentially. Fix $q \in$ $\left(p, p s_{0} /\left(s_{0}-1\right)\right)$ and write $r=q /(q-p)$. We can choose a number $s$ such that $s_{0}<s<r$. Let $X$ denote the set of all $g \in \mathcal{S}(\mathbb{R})$ with $\int_{0}^{\infty} g^{r}(\rho) \rho^{n-1} d \rho \leq 1$. By changes of variables, one has

$$
\begin{aligned}
& \|T f\|_{L_{|x|}^{q} L_{\theta}^{p}\left(\mathbb{R}^{n}\right)}^{p} \\
& =\left(\int_{0}^{\infty}\left(\int_{S^{n-1}}|T f(\rho \theta)|^{p} d \sigma(\theta)\right)^{q / p} \rho^{n-1} d \rho\right)^{p / q} \\
& =\sup _{g \in X} \int_{0}^{\infty} \int_{S^{n-1}}|T f(\rho \theta)|^{p} g(\rho) \rho^{n-1} d \sigma(\theta) d \rho \\
& =\sup _{g \in X} \int_{\mathbb{R}^{n}}|T f(x)|^{p} g(|x|) d x .
\end{aligned}
$$


Fix $g \in X$. Let $I(g):=\int_{\mathbb{R}^{n}}|T f(x)|^{p} g(|x|) d x$ and $h(x)=$ $g(|x|)$. By (45), Hölder's inequality, and changes of variables,

$$
\begin{aligned}
& I(g) \leq C_{p, s, s_{0}} \int_{\mathbb{R}^{n}}|f(x)|^{p} \mathscr{G}_{s}(h)(x) d x \\
& \leq C_{p, s, s_{0}} \int_{0}^{\infty} \int_{S^{n-1}}|f(\rho \theta)|^{p} d \sigma(\theta) \mathscr{G}_{s}(g)(\rho) \rho^{n-1} d \rho \\
& \left.\leq C_{p, s, s_{0}} \int_{0}^{\infty}\left(\int_{S^{n-1}}|f(\rho \theta)|^{p} d \sigma(\theta)\right)^{q / p} \rho^{n-1} d \rho\right)^{p / q} \\
& \quad \times\left(\int_{0}^{\infty}\left(\mathscr{G}_{s}(g)(\rho)\right)^{r} \rho^{n-1} d \rho\right)^{1 / r} \\
& \leq C_{p, q}\|f\|_{L_{|x|}^{q} L_{\theta}^{p}\left(\mathbb{R}^{n}\right)}^{p}\left\|\mathscr{G}_{s}(h)\right\|_{L^{r}\left(\mathbb{R}^{n}\right)} \\
& \leq C_{p, q}\|f\|_{L_{|x|}^{q} L_{\theta}^{p}\left(\mathbb{R}^{n}\right)},
\end{aligned}
$$

which together with (49) leads to (46).

\section{Proofs of Theorems 1 and 2}

We begin with the proof of Theorem 1.

Proof of Theorem 1. By the duality we can write

$$
\mathcal{S}_{\Omega, P_{N}} f(x)=\left(\int_{0}^{\infty}\left|\sigma_{N, r} * f(x)\right|^{2} \frac{d r}{r}\right)^{1 / 2} .
$$

Let $\phi$ be a nonnegative Schwartz function supported in $\{|t| \leq 1\}$ satisfying $\phi(t)=1$ when $|t|<1 / 2$. For $1 \leq \lambda \leq N$, we define the Borel measures $\left\{\mu_{\lambda, r}\right\}_{r>0}$ on $\mathbb{R}^{n}$ by

$$
\begin{aligned}
\widehat{\mu_{\lambda, r}}(\xi)= & \widehat{\sigma_{\lambda, r}}(\xi) \prod_{j=\lambda+1}^{N} \phi\left(\left|r^{j} a_{j} \xi\right|\right) \\
& -\widehat{\sigma_{\lambda-1, r}}(\xi) \prod_{j=\lambda}^{N} \phi\left(\left|r^{j} a_{j} \xi\right|\right), \quad \forall \xi \in \mathbb{R}^{n} .
\end{aligned}
$$

One can verify that

$$
\begin{aligned}
& \sigma_{N, r}=\sum_{\lambda=1}^{N} \mu_{\lambda, r} ; \\
& \int_{2^{k}}^{2^{k+1}}\left|\widehat{\mu_{\lambda, r}}(\xi)\right|^{2} \frac{d r}{r} \\
& \quad \leq C_{\Omega, q}\left(\min \left\{1,\left|2^{k \lambda} a_{\lambda} \xi\right|,\left|2^{k \lambda} a_{\lambda} \xi\right|^{-1}\right\}\right)^{1 /\left(2 q^{\prime} \lambda\right)} ; \\
& M_{\lambda}^{\mu} f(x) \leq M^{N-\lambda} M_{\lambda}^{\sigma}|f|(x)+M^{N-\lambda+1} M_{\lambda-1}^{\sigma}|f|(x)
\end{aligned}
$$

Equation (51) together with (53) and Minkowski's inequality yields that

$$
\begin{aligned}
\mathcal{S}_{\Omega, P_{N}} f(x) & \leq \sum_{\lambda=1}^{N}\left(\int_{0}^{\infty}\left|\mu_{\lambda, r} * f(x)\right|^{2} \frac{d r}{r}\right)^{1 / 2} \\
& =: \sum_{\lambda=1}^{N} T_{\lambda} f(x) .
\end{aligned}
$$

It follows from [31] that $u \leq \mathrm{M}_{s} u$ and $\mathrm{M}_{s} u \in A_{1}$ for $s>1$. From (55) we have

$$
\begin{aligned}
& \sum_{\lambda=1}^{N} \mathrm{M}_{s} M_{\lambda, s}^{\widetilde{\mu}} \mathrm{M}_{s} u \\
& \quad \leq \sum_{\lambda=1}^{N}\left(\mathrm{M}_{s}^{N+1-\lambda} M_{\lambda, s}^{\widetilde{\sigma}} \mathrm{M}_{s} u+\mathrm{M}_{s}^{N+2-\lambda} M_{\lambda-1, s}^{\widetilde{\sigma}} \mathrm{M}_{s} u\right) \\
& \quad \leq \Lambda_{N, s} u .
\end{aligned}
$$

By (56) and (57), to prove Theorem 1, it suffices to show that

$$
\left\|T_{\lambda} f\right\|_{L^{p}(u)} \leq C_{\Omega, q, p, \lambda, s}\|f\|_{L^{p}\left(M_{s} M_{\lambda, s}^{\tilde{\mu}} u\right.}
$$

holds for all $1 \leq \lambda \leq N$ and any nonnegative measurable function $u$ on $\mathbb{R}^{n}$, provided that one of the following conditions holds:

(i) $1<p<2$ and $s>2 / p$;

(ii) $2 \leq p<\infty$ and $s>1$.

We now prove (58) for the case $1<p<2$ and $s>2 / p$. For $1 \leq \lambda \leq N$, let $\Psi_{\lambda}(t) \in \mathscr{C}_{c}^{\infty}((1 / 4,1))$ such that $0 \leq \Psi_{\lambda} \leq 1$ and $\sum_{k \in \mathbb{Z}}\left(\Psi_{\lambda}\left(2^{k \lambda}\left|a_{\lambda} \xi\right|\right)\right)^{3}=1$. Define the Fourier multiplier operators $\left\{\Gamma_{k, \lambda}\right\}_{k \in \mathbb{Z}}$ by $\Gamma_{k, \lambda} f(x)=\Theta_{k, \lambda} * f(x)$, where $\widehat{\Theta_{k, \lambda}}(\xi)=$ $\Psi_{\lambda}\left(2^{k \lambda}\left|a_{\lambda} \xi\right|\right)$. It was shown in [28] that

$$
\left\|\left(\sum_{k \in \mathbb{Z}}\left|\Gamma_{k, \lambda} f\right|^{2}\right)^{1 / 2}\right\|_{L^{p}(w)} \leq C_{p, w, \lambda}\|f\|_{L^{p}(w)}
$$

and

$$
\left\|\sum_{k \in \mathbb{Z}} \Gamma_{k, \lambda} f_{k}\right\|_{L^{p}(w)} \leq C_{p, w, \lambda}\left\|\left(\sum_{k \in \mathbb{Z}}\left|f_{k}\right|^{2}\right)^{1 / 2}\right\|_{L^{p}(w)}
$$

for all $1<p<\infty$ and $w \in A_{p}$.

By the changes of variables and Minkowski's inequality, we can write

$$
\begin{gathered}
T_{\lambda} f(x)=\left(\sum_{k \in \mathbb{Z}} \int_{2^{k}}^{2^{k+1}}\left|\mu_{\lambda, r} * f(x)\right|^{2} \frac{d r}{r}\right)^{1 / 2} \\
=\left(\sum_{k \in \mathbb{Z}} \int_{1}^{2}\left|\mu_{\lambda, 2^{k} r} * f(x)\right|^{2} \frac{d r}{r}\right)^{1 / 2} \\
=\left(\sum_{k \in \mathbb{Z}} \int_{1}^{2}\left|\sum_{j \in \mathbb{Z}} \Gamma_{j+k, \lambda}^{3}\left(\mu_{\lambda, 2^{k} r} * f\right)(x)\right|^{2} \frac{d r}{r}\right)^{1 / 2} \\
\leq \sum_{j \in \mathbb{Z}}\left(\sum_{k \in \mathbb{Z}} \int_{1}^{2}\left|\Gamma_{j+k, \lambda}^{3}\left(\mu_{\lambda, 2^{k} r} * f\right)(x)\right|^{2} \frac{d r}{r}\right)^{1 / 2} \\
=: \sum_{j \in \mathbb{Z}} T_{\lambda, j} f(x) .
\end{gathered}
$$

Hence, by (61), to prove (58) for the case $1<p<2$ and $s>2 / p$, it suffices to show that for any $1<p<2$ and $s>2 / p$, there exists $\alpha(p, q, \gamma)>0$ independent of $j$ such that

$$
\left\|T_{\lambda, j} f\right\|_{L^{p}(u)} \leq C_{\Omega, q, p, \lambda} 2^{-\alpha(p, q, \gamma)|j|}\|f\|_{L^{p}\left(\mathrm{M}_{s} M_{\lambda, s}^{\tilde{\mu}} u\right)} .
$$


We now prove (62). Fix a nonnegative measurable function $u$ on $\mathbb{R}^{n}$. By (54) and Plancherel's theorem, we have

$$
\begin{gathered}
\int_{\mathbb{R}^{n}} \int_{1}^{2}\left|\mu_{\lambda, 2^{k} r} * \Gamma_{j+k, \lambda} w(x)\right|^{2} \frac{d r}{r} d x \\
\leq C_{\Omega, q^{2}} 2^{-|j| / 2 q^{\prime} \gamma^{\prime}} \int_{\mathbb{R}^{n}}|w(x)|^{2} d x
\end{gathered}
$$

for arbitrary function $w$ on $\mathbb{R}^{n}$. One can easily check that

$$
\begin{aligned}
& \int_{\mathbb{R}^{n}} \int_{1}^{2}\left|\mu_{\lambda, 2^{k} r} * \Gamma_{j+k, \lambda} w(x)\right|^{2} \frac{d r}{r} u^{s}(x) d x \\
& \quad \leq \sup _{r>0}\left\|\mu_{\lambda, r}\right\|\left\|\Theta_{j+k, \lambda}\right\|_{L^{1}\left(\mathbb{R}^{n}\right)} \\
& \quad \cdot \int_{1}^{2} \int_{\mathbb{R}^{n}}\left|\mu_{\lambda, 2^{k} r}\right| *\left|\Theta_{j+k, \lambda}\right| *|w|^{2}(x) u^{s}(x) d x \frac{d r}{r} \\
& \leq C_{\Omega, q} \int_{\mathbb{R}^{n}}|w(x)|^{2} \mathrm{MM}_{\lambda}^{\widetilde{\mu}} u^{s}(x) d x
\end{aligned}
$$

for any $s>1$. By (63)-(64) and the interpolation of $L^{2}$-spaces with change of measure ([32, Theorem 5.4.1]), we obtain

$$
\begin{aligned}
& \int_{\mathbb{R}^{n}} \int_{1}^{2}\left|\mu_{\lambda, 2^{k} r} * \Gamma_{j+k, \lambda} w(x)\right|^{2} \frac{d r}{r} u(x) d x \\
& \leq C_{\Omega, q, s} 2^{-\left((1-1 / s) / 2 q^{\prime} \gamma^{\prime}\right)|j|} \int_{\mathbb{R}^{n}}|w(x)|^{2} \mathrm{M}_{s} M_{\lambda, s}^{\tilde{\mu}} u(x) d x
\end{aligned}
$$

for any $s>1$. By (65) with $w=\Gamma_{j+k, \lambda} f$, (60), and the wellknown property of the Rademacher's function, we obtain

$$
\begin{aligned}
& \left\|T_{\lambda, j} f\right\|_{L^{2}(u)}^{2} \\
& =\int_{\mathbb{R}^{n}} \sum_{k \in \mathbb{Z}} \int_{1}^{2}\left|\Gamma_{j+k, \lambda}^{3} \mu_{\lambda, 2^{k} r} * f(x)\right|^{2} \frac{d r}{r} u(x) d x \\
& =\sum_{k \in \mathbb{Z}} \int_{1}^{2} \int_{\mathbb{R}^{n}}\left|\Gamma_{j+k, \lambda}^{3} \mu_{\lambda, 2^{k} r} * f(x)\right|^{2} u(x) d x \frac{d r}{r} \\
& \leq C_{\lambda} \sum_{k \in \mathbb{Z}} \int_{1}^{2} \int_{\mathbb{R}^{n}}\left|\mu_{\lambda, 2^{k} r} * \Gamma_{j+k, \lambda}^{2} f(x)\right|^{2} u(x) d x \frac{d r}{r} \\
& \leq C_{\Omega, q, \lambda, s} 2^{-\left((1-1 / s) / 2 q^{\prime} \gamma^{\prime}\right)|j|} \int_{\mathbb{R}^{n}} \sum_{k \in \mathbb{Z}}\left|\Gamma_{j+k, \lambda} f(x)\right|^{2} \\
& \cdot \mathrm{M}_{s} M_{\lambda, s}^{\widetilde{\mu}} u(x) d x \\
& \leq C_{\Omega, q, \lambda, s} 2^{-\left((1-1 / s) / 2 q^{\prime} \gamma^{\prime}\right)|j|}\|f\|_{L^{2}\left(\mathrm{M}_{s} M_{\lambda, s}^{\tilde{\mu}} u\right)}^{2} .
\end{aligned}
$$

It follows that

$$
\left\|T_{\lambda, j} f\right\|_{L^{2}(u)} \leq C_{\Omega, q, \lambda, s} 2^{-\left((1-1 / s) / 4 q^{\prime} \gamma^{\prime}\right)|j|}\|f\|_{L^{2}\left(\mathrm{M}_{s} M_{\lambda, s}^{\tilde{\mu}} u\right)}
$$

for any $s>1$.

On the other hand, fix $k \in \mathbb{Z}$, and it is easy to see that

$$
\begin{array}{r}
\sup _{r>0}\left\|\mu_{\lambda, 2^{k} r} * f\right\|_{L^{\infty}\left(\mathbb{R}^{n}\right)} \leq C_{\Omega, q}\|f\|_{L^{\infty}\left(\mathbb{R}^{n}\right)} ; \\
\int_{\mathbb{R}^{n}} \int_{1}^{2}\left|\mu_{\lambda, 2^{k} r} * f(x)\right| \frac{d r}{r} u(x) d x \leq\|f\|_{L^{1}\left(M_{\lambda}^{\tilde{\mu}} u\right)} .
\end{array}
$$

The interpolation between (68) and (69) implies that

$$
\begin{aligned}
& \int_{\mathbb{R}^{n}} \int_{1}^{2}\left|\mu_{\lambda, 2^{k} r} * f_{k}(x)\right|^{p} \frac{d r}{r} u(x) d x \\
& \quad \leq C_{\Omega, q, p} \int_{\mathbb{R}^{n}}\left|f_{k}(x)\right|^{p} M_{\lambda}^{\widetilde{\mu}} u(x) d x
\end{aligned}
$$

for all $1<p<2$. It follows from (70) that

$$
\begin{gathered}
\int_{\mathbb{R}^{n}} \sum_{k \in \mathbb{Z}} \int_{1}^{2}\left|\mu_{\lambda, 2^{k} r} * f_{k}(x)\right|^{p} \frac{d r}{r} u(x) d x \\
\leq C_{\Omega, q, p} \int_{\mathbb{R}^{n}} \sum_{k \in \mathbb{Z}}\left|f_{k}(x)\right|^{p} M_{\lambda}^{\widetilde{\mu}} u(x) d x
\end{gathered}
$$

for all $1<p<2$. On the other hand, we have

$$
\begin{gathered}
\int_{\mathbb{R}^{n}}\left(\sup _{k \in \mathbb{Z} r \in[1,2]} \sup _{r, 2^{k} r} * f_{k}(x) \mid\right)^{p} d x \\
\leq C_{\Omega, q, p} \int_{\mathbb{R}^{n}}\left(\sup _{k \in \mathbb{Z}}\left|f_{k}(x)\right|\right)^{p} d x
\end{gathered}
$$

for all $1<p<2$. By interpolating between (71) and (72),

$$
\begin{aligned}
& \int_{\mathbb{R}^{n}}\left(\sum_{k \in \mathbb{Z}} \int_{1}^{2}\left|\mu_{\lambda, 2^{k} r} * f_{k}(x)\right|^{2} \frac{d r}{r}\right)^{p / 2} u^{1 / t_{1}}(x) d x \\
& \leq C_{\Omega, q, p, t_{1}} \int_{\mathbb{R}^{n}}\left(\sum_{k \in \mathbb{Z}}\left|f_{k}(x)\right|^{2}\right)^{p / 2}\left(M_{\lambda}^{\widetilde{\mu}} u\right)^{1 / t_{1}}(x) d x
\end{aligned}
$$

for all $1<p<2$, where $t_{1}=2 / p$. Substituting $u^{t_{1}}$ for $u$ in (73), we obtain

$$
\begin{aligned}
& \int_{\mathbb{R}^{n}}\left(\sum_{k \in \mathbb{Z}} \int_{1}^{2}\left|\mu_{\lambda, 2^{k} r} * f_{k}(x)\right|^{2} \frac{d r}{r}\right)^{p / 2} u(x) d x \\
& \leq C_{\Omega, q, p, t_{1}} \int_{\mathbb{R}^{n}}\left(\sum_{k \in \mathbb{Z}}\left|f_{k}(x)\right|^{2}\right)^{p / 2}\left(M_{\lambda, t_{1}}^{\widetilde{\mu}} u\right)(x) d x .
\end{aligned}
$$

Since $M_{\lambda, t_{1}}^{\widetilde{\mu}} u \in A_{1}$, by the weighted Littlewood-Paley theory and (74),

$$
\begin{aligned}
& \left\|T_{\lambda, j} f\right\|_{L^{p}(u)} \\
& =\left\|\left(\sum_{k \in \mathbb{Z}} \int_{1}^{2}\left|\mu_{\lambda, 2^{k} r} * \Gamma_{j+k, \lambda}^{3} f\right|^{2} \frac{d r}{r}\right)^{1 / 2}\right\|_{L^{p}(u)} \\
& \leq C_{\Omega, q, p, t_{1}}\left\|\left(\sum_{k \in \mathbb{Z}}\left|\Gamma_{j+k, \lambda}^{3} f\right|^{2}\right)^{1 / 2}\right\| \\
& \quad \leq C_{\Omega, q, p, \lambda, t_{1}}\|f\|_{L^{p}\left(M_{\lambda, t_{1}}^{\tilde{\mu}} u\right)}
\end{aligned}
$$


for all $1<p<2$. Letting $s=t_{1}$, (67) gives us that

$$
\begin{aligned}
& \left\|T_{\lambda, j} f\right\|_{L^{2}(u)} \\
& \quad \leq C_{\Omega, q, \lambda, t_{1}} 2^{-\left(\left(1-1 / t_{1}\right) / 4 q^{\prime} \gamma^{\prime}\right)|j|}\|f\|_{L^{2}\left(\mathrm{M}_{t_{1}} M_{\lambda, t_{1}}^{\widetilde{\mu}} u\right)} .
\end{aligned}
$$

By an interpolation between (75) and (76), one has

$$
\left\|T_{\lambda, j} f\right\|_{L^{p}(u)} \leq C_{\Omega, q, p, \lambda} 2^{-\alpha(p, q, \gamma)|j|}\|f\|_{L^{p}\left(\mathrm{M}_{t_{1}} M_{\lambda, t_{1}}^{\tilde{\mu}} u\right)}
$$

for all $1<p<2$, where $\alpha(p, q, \gamma)>0$. Note that $M_{t_{1}} M_{\lambda, t_{1}}^{\widetilde{\mu}} u \leq$ $C M_{s} M_{\lambda, s}^{\widetilde{\mu}} u$ for $s>t_{1}$ by Hölder's inequality. This together with (61) yields (58).

It remains to show (58) for the case $2 \leq p<\infty$ and $s>1$. We want to show that

$$
\left\|T_{\lambda, j} f\right\|_{L^{p}(u)} \leq C_{\Omega, q, p, \lambda, s}\|f\|_{L^{p}\left(\mathrm{M}_{s} M_{\lambda, s}^{\tilde{\mu}} u\right)}
$$

for all $2<p<\infty$. Actually, by (67), (78), and an interpolation (see [32, Corollary 5.5.4]), one has

$$
\left\|T_{\lambda, j} f\right\|_{L^{p}(u)} \leq C_{\Omega, q, p, \lambda, s^{-\beta(p, q, \gamma, s)|j|}} 2^{-f} \|_{L^{p}\left(\mathrm{M}_{s} M_{\lambda, s}^{\widetilde{\mu}} u\right)}
$$

for $2 \leq p<\infty$ and $s>1$. Here $\beta(p, q, \gamma, s)>0$ depends only on $p, q, \gamma$, and $s$. Combining (79) with (61) yields (58) for the case $2 \leq p<\infty$ and $s>1$.

Next we shall prove (78). Fix $2<p<\infty$. By duality we can choose a function $v \in L^{(p / 2)^{\prime}}(u)$ with unit norm such that

$$
\begin{aligned}
& \left\|\left(\sum_{k \in \mathbb{Z}} \int_{1}^{2}\left|\mu_{\lambda, 2^{k} r} * g_{k}\right|^{2} \frac{d r}{r}\right)^{1 / 2}\right\|_{L^{p}(u)}^{2} \\
& =\int_{\mathbb{R}^{n}} \sum_{k \in \mathbb{Z}} \int_{1}^{2}\left|\mu_{\lambda, 2^{k} r} * g_{k}(x)\right|^{2} \frac{d r}{r} \cdot v(x) u(x) d x .
\end{aligned}
$$

This together with the fact that $\left\|\mu_{k, t}^{\lambda}\right\| \leq C_{\Omega, q}$ shows that

$$
\begin{aligned}
& \left\|\left(\sum_{k \in \mathbb{Z}} \int_{1}^{2}\left|\mu_{\lambda, 2^{k} r} * g_{k}\right|^{2} \frac{d r}{r}\right)^{1 / 2}\right\|_{L^{p}(u)}^{2} \\
& \leq C_{\Omega, q} \int_{\mathbb{R}^{n}} \sum_{k \in \mathbb{Z}}\left|g_{k}(x)\right|^{2} \int_{1}^{2}|| \tilde{\mu}_{\lambda, 2^{k} r}|*(v u)(x)| \frac{d r}{r} d x .
\end{aligned}
$$

Fix $s>1$ and let $t=p s / 2$. By Hölder's inequality,

$$
\begin{aligned}
& || \widetilde{\mu}_{\lambda, 2^{k_{r}}}|*(v u)| \\
& \quad \leq\left(\left|\widetilde{\mu}_{\lambda, 2^{k} r}\right| * u^{s}\right)^{1 / t}\left(\left|\widetilde{\mu}_{\lambda, 2^{k} r}\right| *\left(u^{t^{\prime} /(p / 2)^{\prime}} v^{t^{\prime}}\right)\right)^{1 / t^{\prime}} .
\end{aligned}
$$

By Hölder's inequality with exponents $p / 2$ and $(p / 2)^{\prime}$ again and (81)-(82),

$$
\begin{gathered}
\left\|\left(\sum_{k \in \mathbb{Z}} \int_{1}^{2}\left|\mu_{\lambda, 2^{k} r} * g_{k}\right|^{2} \frac{d t}{t}\right)^{1 / 2}\right\|_{L^{p}(u)}^{2} \\
\leq C_{h, \Omega, q, \gamma} \int_{\mathbb{R}^{n}} \sum_{k \in \mathbb{Z}}\left|g_{k}(x)\right|^{2}\left(M_{\lambda}^{\widetilde{\mu}} u^{s}\right)^{1 / t} \\
\left.\cdot M_{\lambda}^{\widetilde{\mu}}\left(u^{t^{\prime} /(p / 2)^{\prime}} v^{t^{\prime}}\right)\right)^{1 / t^{\prime}}(x) d x \\
\leq C_{h, \Omega, q, \gamma}\left\|\left(\sum_{k \in \mathbb{Z}}\left|g_{k}\right|^{2}\right)^{1 / 2}\right\|_{L^{p}\left(M_{\lambda, s}^{\tilde{\mu}} u\right)}^{2} \\
\cdot\left\|M_{\lambda}^{\widetilde{\mu}}\left(u^{t^{\prime} /(p / 2)^{\prime}} v^{t^{\prime}}\right)\right\|_{L^{(p / 2)^{\prime} / t^{\prime}}\left(\mathbb{R}^{n}\right)}^{1 / t^{\prime}} .
\end{gathered}
$$

On the other hand, by Lemma 6 and (55), we have

$$
\left\|M_{\lambda}^{\widetilde{\mu}} f\right\|_{L^{r}\left(\mathbb{R}^{n}\right)} \leq C_{h, \Omega, q, \gamma, r}\|f\|_{L^{r}\left(\mathbb{R}^{n}\right)}, \quad \forall 1<r<\infty .
$$

Since $p / 2=t / s<t$, then $(p / 2)^{\prime}>t^{\prime}$. (84) leads to

$$
\begin{aligned}
& \left\|M_{\lambda}^{\widetilde{\mu}}\left(u^{t^{\prime} /(p / 2)^{\prime}} v^{t^{\prime}}\right)\right\|_{L^{(p / 2)^{\prime} / t^{\prime}}\left(\mathbb{R}^{n}\right)}^{1 / t^{\prime}} \\
& \quad \leq C_{h, \Omega, q, \gamma, p, s}\left\|u^{t^{\prime} /(p / 2)^{\prime}} v^{t^{\prime}}\right\|_{L^{(p / 2)^{\prime} / t^{\prime}}\left(\mathbb{R}^{n}\right)}^{1 / t^{\prime}} \leq C_{\Omega, q, p, s} .
\end{aligned}
$$

This together with (84) yields that

$$
\begin{gathered}
\left\|\left(\sum_{k \in \mathbb{Z}} \int_{1}^{2}\left|\mu_{\lambda, 2^{k} r} * g_{k}\right|^{2} \frac{d r}{r}\right)^{1 / 2}\right\|_{L^{p}(u)} \\
\leq C_{\Omega, q, p, s}\left\|\left(\sum_{k \in \mathbb{Z}}\left|g_{k}\right|^{2}\right)^{1 / 2}\right\| \|_{L^{p}\left(M_{\lambda, s}^{\tilde{\mu}} u\right)}
\end{gathered}
$$

for all $2<p<\infty$ and any $s>1$.

It follows from (59), (60), (86), and the fact that $M_{\lambda, s}^{\widetilde{\mu}} u \leq$ $\mathrm{M}_{s} M_{\lambda, s}^{\widetilde{\mu}} u$ that

$$
\begin{aligned}
& \left\|T_{\lambda, j} f\right\|_{L^{p}(u)} \\
& =\left\|\left(\sum_{k \in \mathbb{Z}} \int_{1}^{2}\left|\mu_{\lambda, 2^{k} r} * \Gamma_{j+k, \lambda}^{3} f\right|^{2} \frac{d r}{r}\right)^{1 / 2}\right\|_{L^{p}(u)} \\
& \leq C_{\Omega, q, p, \lambda, s}\left\|\left(\sum_{k \in \mathbb{Z}}\left|\Gamma_{j+k, \lambda}^{3} f\right|^{2}\right)^{1 / 2}\right\| \\
& \leq C_{\Omega, q, p, \lambda, s}\|f\|_{L^{p}\left(\mathrm{M}_{s} M_{\lambda, s}^{\tilde{\mu}} u\right)}
\end{aligned}
$$

for all $2<p<\infty$ and any $s>1$. This gives (78) and completes the proof of Theorem 1 . 
Proof of Theorem 2. By Lemma 6, one has

$$
\left\|\Lambda_{N, s} u\right\|_{L^{r}\left(\mathbb{R}^{n}\right)} \leq C_{h, \Omega, q, \gamma}\|u\|_{L^{r}\left(\mathbb{R}^{n}\right)}
$$

for any $r>s$. By (88), Theorem 1, and Proposition 7, we obtain (16)-(18) for the case $1<\tilde{p}<2$ and $\tilde{p}<p<2 \widetilde{p} /(2-\tilde{p})$ or $2 \leq \widetilde{p}<p<\infty$. It was known that $\mathrm{M}_{\Omega, P_{N}}$ is bounded on $L^{p}\left(\mathbb{R}^{n}\right)$ for $2 \leq p<\infty$. This together with (7) yields (16)-(18) for the case of $2 \leq p=\widetilde{p}<\infty$.

\section{Data Availability}

No data were used to support this study.

\section{Conflicts of Interest}

The authors declare that there are no conflicts of interest regarding the publication of this paper.

\section{Authors' Contributions}

The authors read and approved the final manuscript.

\section{Acknowledgments}

The first author was partially supported by the NNSF of China (Grant no. 11701333) and Support Program for Outstanding Young Scientific and Technological Top-notch Talents of College of Mathematics and Systems Science (Grant no. Sxy2016K01). The second author was partially supported by Shandong Natural Science Foundation (Grant no. ZR2019QA017).

\section{References}

[1] F. Liu, "A note on Marcinkiewicz integrals associated to surfaces of revolution," Journal of the Australian Mathematical Society, vol. 104, no. 3, pp. 380-420, 2018.

[2] F. Liu, "Boundedness and continuity of maximal operators associated to polynomial compound curves on Triebel-Lizorkin spaces," Mathematical Inequalities \& Applications, no. 1, pp. 2544, 1998.

[3] F. Liu, "Rough maximal functions supported by subvarieties on Triebel-Lizorkin spaces," Revista de la Real Academia de Ciencias Exactas, Físicas y Naturales. Serie A. Matemáticas, vol. 112, no. 2, pp. 593-614, 2018.

[4] F. Liu, S. Mao, and H. Wu, "On rough singular integrals related to homogeneous mappings," Collectanea Mathematica, vol. 67, no. 1, pp. 113-132, 2016.

[5] F. Liu and H. Wu, "Lp bounds for Marcinkiewicz integrals associated to homogeneous mappings," Monatshefte für Mathematik, vol. 181, no. 4, pp. 875-906, 2016.

[6] F. Liu and H. Wu, "A note on the endpoint regularity of the discrete maximal operator," Proc. Amer. Math. Soc, vol. 147, no. 2, pp. 583-596, 219.

[7] F. Liu, Q. Xue, and K. Yabuta, "Rough maximal singular integral and maximal operators supported by subvarieties on Triebel-Lizorkin spaces," Nonlinear Analysis. Theory, Methods \& Applications. An International Multidisciplinary Journal, vol. 171, pp. 41-72, 2018.
[8] F. Liu, Q. Xue, and K. Yabuta, "Boundedness and continuity of maximal singular integrals and maximal functions on TriebelLizorkin spaces," Science China-Mathematics, In press.

[9] F. Cacciafesta and P. D'Ancona, "Endpoint estimates and global existence for the nonlinear Dirac equation with potential," Journal of Differential Equations, vol. 254, no. 5, pp. 2233-2260, 2013.

[10] Y. Gu, C.-M. Fan, and R.-P. Xu, "Localized method of fundamental solutions for large-scale modeling of two-dimensional elasticity problems," Applied Mathematics Letters, vol. 93, pp. 814, 2019.

[11] S. Machihara, M. Nakamura, K. Nakanishi, and T. Ozawa, "Endpoint Strichartz estimates and global solutions for the nonlinear Dirac equation," Journal of Functional Analysis, vol. 219, no. 1, pp. 1-20, 2005.

[12] J. Sterbenz, "Angular regularity and Strichatz estimates for the wave equation," International Mathematics Research Notices, vol. 4, pp. 187-231, 2005.

[13] T. Tao, "Spherically averaged endpoint Strichartz estimates for the two-dimensional Schrödinger equation," Communications in Partial Differential Equations, vol. 25, no. 7-8, pp. 1471-1485, 2000.

[14] S. Xi and S. Zhu, "Blow-up criterion for the 3D non-resistive compressible magnetohydrodynamic equations," Journal of Dynamics and Differential Equations, 2019.

[15] F. Xu and W. Gan, "On a lotka-volterra type competition model from river ecology," Nonlinear Analysis: Real World Applications, vol. 47, pp. 373-384, 2019.

[16] J. Wang, H. Cheng, H. Liu, and Y. Wang, "Periodic solution and control optimization of a prey-predator model with two types of harvesting," Advances in Difference Equations, vol. 2018, 14 pages, 2018.

[17] F. Cacciafesta and R. Lucà, "Singular integrals with angular integrability," Proceedings of the American Mathematical Society, vol. 144, no. 8, pp. 3413-3418, 2016.

[18] A. Córdoba, "Singular integrals, maximal functions and Fourier restriction to spheres: the disk multiplier revisited," Advances in Mathematics, vol. 290, pp. 208-235, 2016.

[19] P. D'Ancona and R. Lucà, "On the Regularity Set and Angular Integrability for the Navier-Stokes Equation," Archive for Rational Mechanics and Analysis, vol. 221, no. 3, pp. 1255-1284, 2016.

[20] F. Liu and D. Fan, "Weighted estimates for rough singular integrals with applications to angular integrability," Pacific Journal of Mathematics, In press.

[21] L. Chen and H. Lin, "A maximal operator related to a class of singular integrals," Illinois Journal of Mathematics, vol. 34, no. 1, pp. 120-126, 1990.

[22] H. M. Al-Qassem, "Maximal operators related to block spaces," Kodai Mathematical Journal, vol. 28, no. 3, pp. 494-510, 2005.

[23] A. Al-Salman, "Rough maximal functions supported by subvarieties," The Journal of Operator Theory, vol. 59, no. 2, pp. 263$275,2008$.

[24] A. Al-Salman, "On maximal functions with rough kernels in L(log+L)1/2(Sn-1)," Collect. Math, vol. 56, no. 1, pp. 47-56, 2005.

[25] A. Al-Salman, "On a Class of Singular Integral Operators With Rough Kernels," Canadian Mathematical Bulletin, vol. 49, no. 1, pp. 3-10, 2006.

[26] J. Zhu, K. Li, and B. Hao, "Image restoration by second-order total generalized variation and wavelet frame regularization," Complexity, vol. 2019, Article ID 3650128, 16 pages, 2019. 
[27] J. Duoandikoetxea and J. L. Rubio de Francia, "Maximal and singular integral operators via Fourier transform estimates," Inventiones Mathematicae, vol. 84, no. 3, pp. 541-561, 1986.

[28] S. Hofmann, "Weighted norm inequalities and vector valued inequalities for certain rough operators," Indiana University Mathematics Journal, vol. 42, no. 1, p. 1, 1993.

[29] F. Liu, Z. Fu, and S. T. Jhang, "Boundedness and continuity of Marcinkiewicz integrals associated to homogeneous mappings on Triebel-Lizorkin spaces," Frontiers of Mathematics in China, vol. 14, no. 1, pp. 95-122, 2019.

[30] F. Ricci and E. M. Stein, "Harmonic analysis on nilpotent groups and singular integrals. I. Oscillatory integrals," Journal of Functional Analysis, vol. 73, no. 1, pp. 179-194, 1987.

[31] R. R. Coifman and R. Rochberg, "Another Characterization of BMO," Proceedings of the American Mathematical Society, vol. 79, no. 2, pp. 249-254, 1980.

[32] J. Bergh and J. Lofstrom, "Interpolation spaces," in An introduction, Grundlehren der Mathematischen Wissenschaften, vol. 223, Springer-Verlag, Berlin, Germany, NY, USA, 1976. 


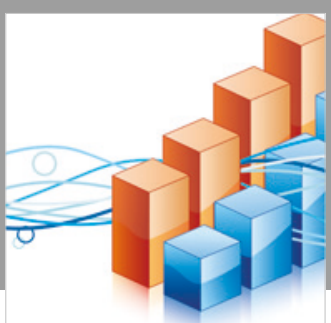

Advances in

Operations Research

\section{-n-m}
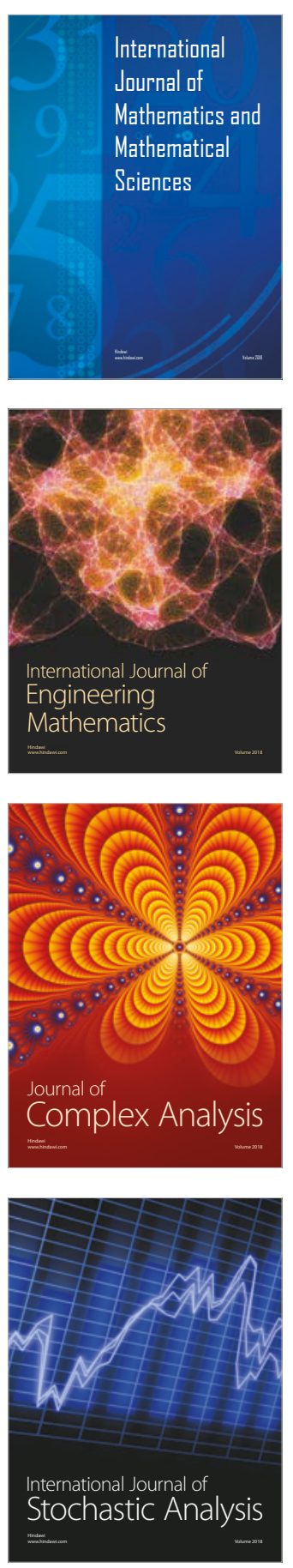
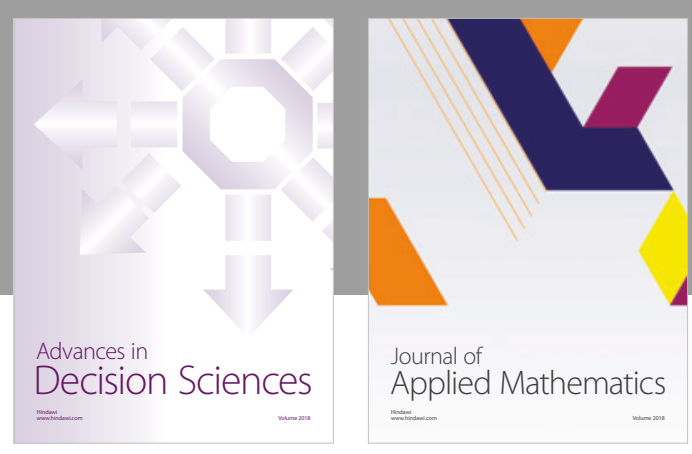

Journal of

Applied Mathematics
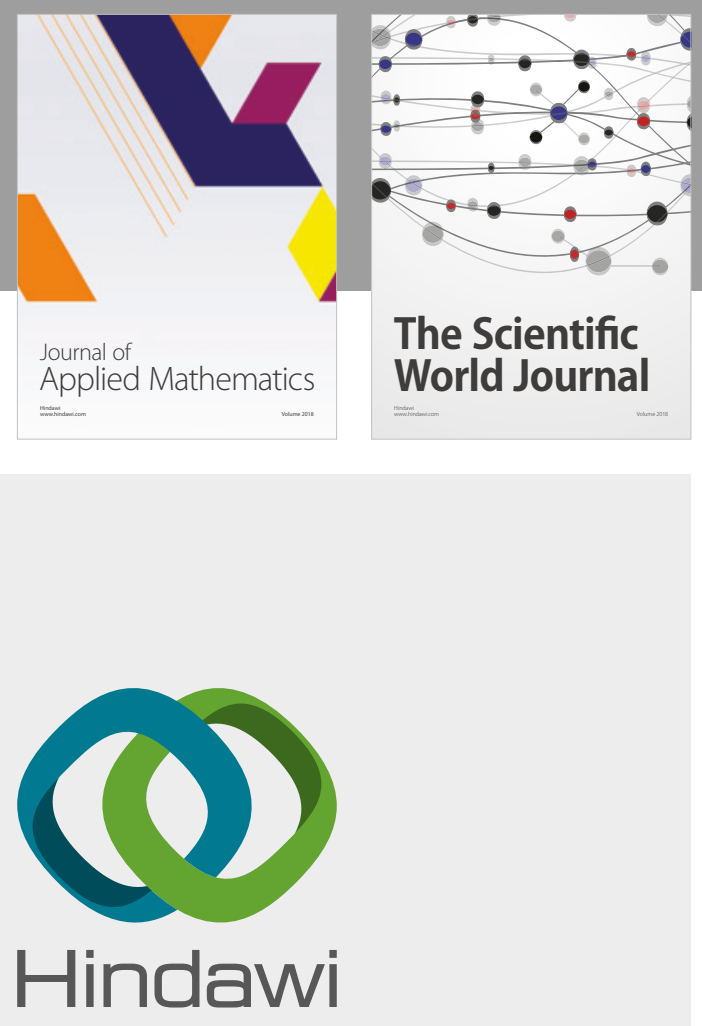

Submit your manuscripts at

www.hindawi.com

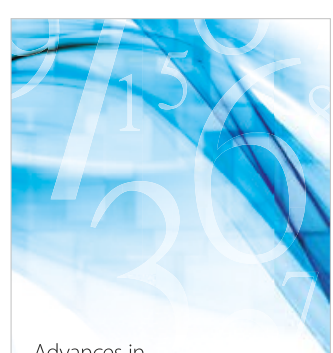

Advances in
Numerical Analysis
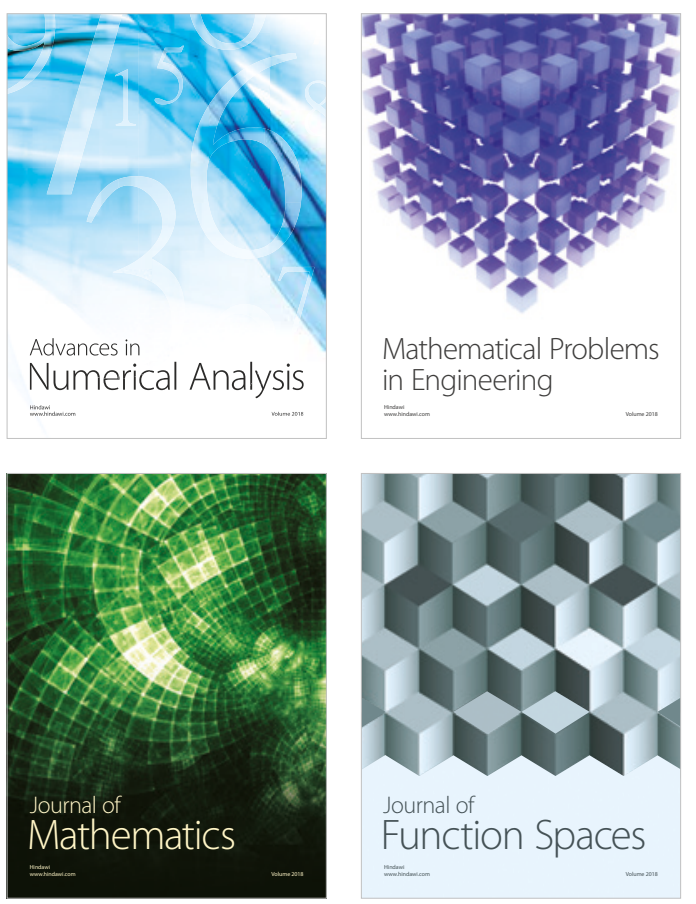

Mathematical Problems in Engineering

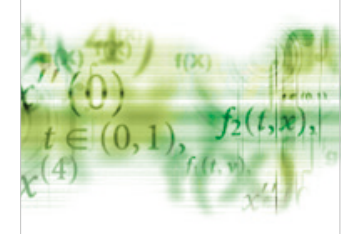

International Journal of

Differential Equations

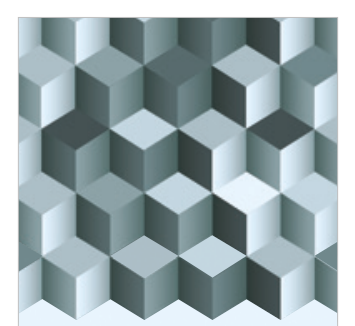

Journal of

Function Spaces

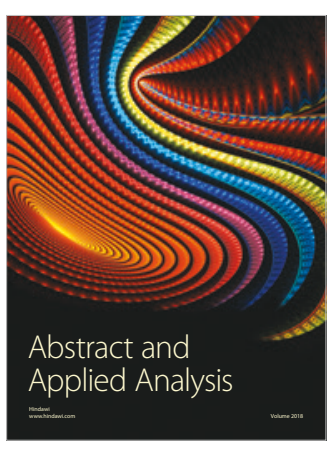

The Scientific

World Journal

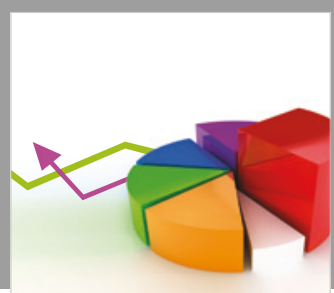

Journal of

Probability and Statistics
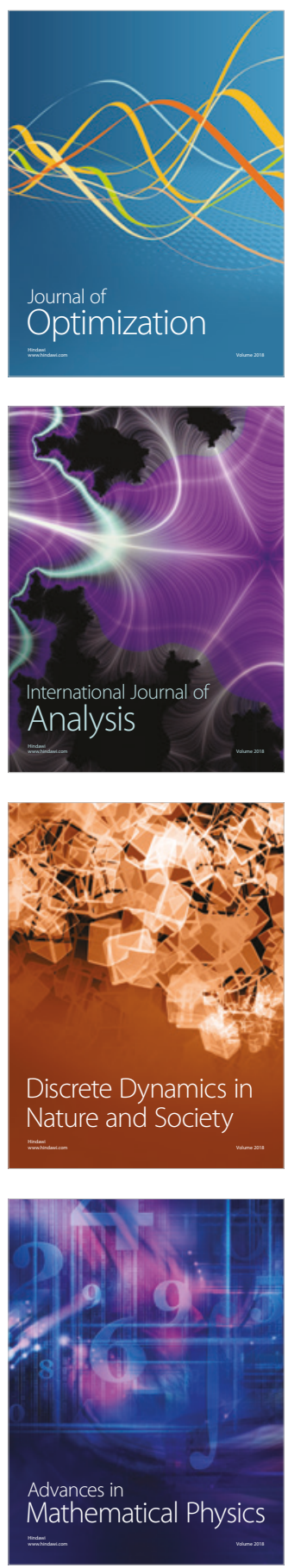